\title{
Implications of Quasi-Geometric Discounting on the Observable Sharpe Ratio
}

Wooheon Rhee

Tack Yun

Wooheon Rhee

Department of Economics

Kyunghee University

1 Hoegi-dong,

Dongdaemoon-gu,

Seoul 130-701, Korea

Tel:82-2-961-0774

E-mail:wrhee@khu.ac.kr

Tack Yun

School of Economics

Kookmin University

861-1, Jeungnung-dong,

Seungbuk-gu,

Seoul 136-702, Korea

Tel:82-2-910-4529

E-mail:yuntack@kmu.kookmin.ac.kr 


\title{
Implications of Quasi-Geometric Discounting on the Observable Sharpe Ratio
}

\begin{abstract}
In this paper, we study quantitative implications of quasi-geometric discounting for stochastic properties of asset returns that can be observed in the financial market data. In particular, we emphasize that the dividend income from an asset measured in a unit of account may not reflect the whole dividend that consumers expect to obtain from the asset in models with quasi-geometric discounting. We then show that allowing for such a possibility in a stochastic growth model with quasi-geometric discounting enables one to match the Sharpe ratio observed in the U.S. data.
\end{abstract}

JEL classification: E32; G12

Keywords: Quasi-Geometric Discounting; Observable and Unobservable Asset Returns; The Sharpe Ratio 


\section{Introduction}

In this paper, we study the role of time-inconsistent preferences on asset pricing in a dynamic stochastic general equilibrium model. The time-inconsistent preferences take place because time discount rates of households are greater or smaller in the short-run than in the long-run, as in the works of $\operatorname{Strotz(1952),~Pollak(1968),~Phelps~and~Pollak(1968),~}$ Laibson(1997), and Krusell and $\operatorname{Smith}(2003 a, b)$.

In particular, we consider a stochastic growth model with quasi-geometric discounting, while state contingent claims on consumption goods are not traded in markets. In such an economic environment, consumers may obtain non-pecuniary payoffs as well as pecuniary payoffs from capital holdings when their time discount rates are greater or smaller in the short-run than in the long-run. The reason for the presence of non-pecuniary payoffs is that when the time inconsistent preferences create strategic interaction between current and future selves of an infinitely-lived consumer, the consumer can restrict his or her future actions through the portfolio choices made at the current period. The strategic interaction therefore may have consumers obtain dividends from their asset holdings, which are not paid by issuers of assets.

The presence of the non-pecuniary payoffs from asset holdings indicates that the observable asset returns may not reflect the whole returns that consumers have from their asset holdings. The break-down of an asset's return into its observable and unobservable components also leads one to construct a measure of risk-return trade-off in terms of the observable return component. In particular, the measure of the risk-return trade-off is defined as the ratio of the risk premium of the observable return component to its conditional standard deviation, which can be interpreted as the observable Sharpe ratio in the context of this paper. We then show that allowing for the presence of unobservable non-pecuniary payoffs helps to match the trade-off between expected return and risk observed in financial data.

The non-pecuniary payoffs from asset holdings are not new in models with quasigeometric discounting. Krusell and $\operatorname{Smith}(2003 \mathrm{~b})$ have shown that the marginal change of the future capital stock in response to the marginal change of current period savings is included in their generalized Euler equation. We interpret this term as the unobservable 
component of the whole investment return, which is absent if consumers with ordinary geometric discount functions have time-consistent preferences. The difference here is that we focus on testable implications for the Sharpe ratio in models with quasi-geometric discounting, which is defined as the slope of the conditional mean-standard deviation frontier.

Shiller(1982) and Hansen and Jaganathan(1991) have shown that the Sharpe ratio can be expressed as a function of the first and second moments of the stochastic discount factor, defined as the intertemporal marginal rate of substitution. In the same vein, we analyze the relationship between the Sharpe ratio and the stochastic discount factor, which is implied by the stochastic growth model with quasi-geometric discounting. The characterization of a link between the Sharpe ratio and the stochastic discount factor necessarily involves a distinction between the stochastic discount factor and asset returns in the Euler equations for asset holdings. In this paper, we define the whole return of an asset as a weighted average of its observable and unobservable returns described above, while the weight for the observable return is set equal to the measure of the short-run impatience of consumers. Given the definition of the whole return of an asset, we show that a candidate for the stochastic discount factor can be written as the intertemporal marginal rate of substitution of consumers who behave as if they discount future utilities at a constant rate.

We also show that the stochastic discount factor defined above turns out to be different from the one used for converting only pecuniary payoffs obtained in the next period into its current period value. The reason for this can be written as follows. In this paper, we show that the generalized Euler equation includes the unobservable return as well as the observable return from capital holdings in the absence of state contingent claims on consumption goods. However, when households trade state contingent claims on consumption goods in complete markets, one can use a no-arbitrage condition to price the capital stock, taking into account only its pecuniary dividends. Therefore, in the presence of state contingent claims, the conditional expectation of the stochastic discount factor times the observable return equals one. As a result, the stochastic discount factor in the presence of state contingent claims differs from the one used for pricing both observable and unobservable returns in the absence of state contingent claims. This explains the reason why our results differ from Luttmer and Mariotti(2003), in which one-period state contingent 
claims on consumption goods are traded in sequentially complete markets.

We now ask if the observable Sharpe ratio generated by a neoclassical stochastic growth model with quasi-geometric discounting can match the Sharpe ratio of the market portfolio observed in the U.S. data. A reason why we are interested in a stochastic growth model is that we can obtain a closed-form solution for the model under a specific parameterization without relying on any approximation to equilibrium conditions. The specific parameterization involves additive separability between consumption and leisure, logarithmic utility for consumption, and complete depreciation of the capital stock within a period, as has been analyzed in Krusell and Smith(2003b).

Lettau and Uhlig(1997a, b) have demonstrated that stochastic growth models do not match the Sharpe ratio observed in the U.S. data, under parameter values that have been widely used in the literature. While our analysis complements their conclusion in terms of the Sharpe ratio of the whole return, we also show that when discount rates of households are greater in the short-run than in the log-run, the observable Sharpe ratio of stochastic growth models can match the Sharpe ratio of the market portfolio observed in the U.S. data. We do this without making the volatility of the intertemporal marginal rate of substitution arbitrarily high.

The reason why quasi-geometric discounting can help modify the standard lower bound of the ratio of the second moment of the stochastic discount factor to its first moment can be explained as follows. While the unobservable whole investment return is a weighted average of observable and unobservable terms, the weight for the observable term decreases as the short-run impatience of households rises. In addition, we show that the unobservable term is less than the observable term in the stochastic growth model we analyze in this paper. Given the observed level of the observable term, it thus means that a rise in the short-run impatience of households leads to a fall in the size of the whole investment return. Hence, when the unobservable Sharpe ratio is defined in terms of the unobservable whole investment return, it falls as the short-run impatience of households rises. As a result, we can find a value of the short-run impatience of household, which allows the unobservable Sharpe ratio to equal the ratio of the second moment of the stochastic discount factor to its first moment given the observed level of the observable term.

Furthermore, we find that the unobservable Sharpe ratio for the competitive equilib- 
rium remains above that of the planner's problem, if discount rates of households are greater in the short-run than in the long-run. This in turn implies that the competitive equilibrium requires a higher value of the short-run impatience of households to match the observed Sharpe ratio than does the planner's problem. The reason for this can be explained as follows. Krusell, Kuruscu and Smith(2002) have shown that price-taking behavior in the competitive equilibrium leads to a higher level of welfare than does the planner's problem. Specifically, when discount rates of households are greater in the shortrun than in the long-run, the price-taking behavior gives the household a higher benefit from extra saving today than does the planner's problem. This in turn implies that the unobservable return from investment is smaller for the planner's problem than for the competitive equilibrium, given the observed level of the observable investment return. Since the whole investment return is a weighted average of observable and unobservable terms, it means that the whole investment return is greater for the competitive equilibrium than for the planner's problem. The unobservable Sharpe ratio is therefore higher for the competitive equilibrium than for the planner's problem. As a result, the competitive equilibrium requires a higher level of short-run impatience of households than does the planner's problem, in order to match the observed Sharpe ratio.

The rest of the paper is organized as follows. In section 2, we analyze closed form solutions for the competitive equilibrium and the planner's problem in a stochastic growth model with quasi- geometric discounting. In section 3, we show that when the whole investment return is defined as a weighted average of observable and unobservable terms, a lower bound of the ratio of the second moment of the stochastic discount factor to its first moment is the Sharpe ratio constructed on the basis of the unobservable whole investment return. We then consider a closed-form relationship between the observable and unobservable Sharpe ratios. We also show that when households are supposed to trade state contingent claims in markets, quasi-geometric discounting does not help match observed levels of the Sharpe ratio. Section 4 concludes. 


\section{Stochastic Growth Models with Quasi-Geometric Discounting}

We begin this section by considering a recursive competitive equilibrium in a stochastic growth model with quasi-geometric discounting and then move onto the discussion of the generalized Euler equation.

\subsection{Competitive Equilibrium}

In this section, we briefly highlight the competitive equilibrium for a stochastic growth model with quasi-geometric discounting. The preference at period $t$ of the representative household is represented by the following utility function:

$$
u\left(C_{t}, 1-H_{t}\right)+\theta \beta\left[\sum_{k=0}^{\infty} \beta^{k} E_{t}\left[u\left(C_{t+1+k}, 1-H_{t+1+k}\right)\right]\right]
$$

where $C_{t}$ is consumption at period $t$ and $H_{t}$ is the hours worked at period $t$. The period utility function $u\left(C_{t}, 1-H_{t}\right)$ is twice differentiable and concave in its arguments.

The household owns the capital stock, which is employed to produce output. In each period $t=0,1, \cdots, \infty$, the capital stock is accumulated according to the following linear technology:

$$
K_{t+1}=I_{t}+(1-\delta) K_{t}
$$

where $\delta$ denotes a constant depreciation rate for capital, $K_{t}$ denotes the capital stock at period $t$, and $I_{t}$ denotes the capital investment at period $t$. Furthermore, letting $\hat{R}_{t}$ denote the real rental rate at period $t$, the observable real return at period $t$ of the capital stock can be written as

$$
R_{t}=1-\delta+\hat{R}_{t}
$$

where $R_{t}$ denotes the observable return component at period $t$ of holding a unit of the capital stock from period $t-1$ through period $t$. The period budget constraint at period $t$ of the household can be written as

$$
C_{t}+K_{t+1}=R_{t} K_{t}+W_{t} H_{t}
$$

where $W_{t}$ denotes the real wage at period $t$. Here, it should be noted that while we may be able to include many different types of assets in the period budget constraint (2.4), 
we allow for only capital accumulation to focus its role on the observable Sharpe ratio. In addition, markets for production inputs and final goods are assumed to be perfectly competitive, so that an individual agent takes their prices as given when the agent makes his or her decision making.

The representative household then maximizes its expected utility function (2.1) subject to the period budget constraint (2.4) in each period $t=0,1, \cdots, \infty$, given an initial value of capital $K_{0}$ and sequences of real prices $\left\{W_{t}, R_{t}\right\}_{t=0}^{\infty}$. The first-order conditions of the representative household's problem are then given by

$$
\begin{gathered}
\frac{u_{2}\left(C_{t}, 1-H_{t}\right)}{u_{1}\left(C_{t}, 1-H_{t}\right)}=W_{t} . \\
1=\beta E_{t}\left[\frac{u_{1}\left(C_{t+1}, 1-H_{t+1}\right)}{u_{1}\left(C_{t}, 1-H_{t}\right)}\left[\theta R_{t+1}+(1-\theta) \frac{\partial K_{t+2}}{\partial K_{t+1}}\right]\right] .
\end{gathered}
$$

Here, equation (2.5) states that the marginal rate of substitution between consumption and leisure equals the real wage. Besides, equation (2.6) corresponds to the generalized Euler equation in a model with quasi-geometric discounting. Appendix A shows how to derive the Euler equation from the optimization of the household.

Furthermore, output from production for firm $i, Y_{i t}$, is determined by a constant returns to scale production function of the form:

$$
Y_{i t}=A_{t} F\left(K_{i t}, H_{i t}\right)
$$

where $A_{t}$ denotes the aggregate random technology disturbances at period $t, K_{i t}$ denotes the capital stock rented by firm $i$, and $H_{i t}$ denotes the number of hours hired by firm $i$. The logarithm of the total factor productivity follows an $\mathrm{AR}(1)$ process of the form:

$$
a_{t}=\rho_{a} a_{t-1}+e_{t}
$$

where $a_{t}=\log A_{t}$ and $e_{t}$ is an i.i.d. random variable with its mean zero and standard deviation $\sigma_{a}$. Hence, the real wage and real rental rates in an equilibrium can be written as

$$
W_{t}=A_{t} F_{2}\left(\bar{K}_{t}, H_{t}\right) ; \quad \hat{R}_{t}=A_{t} F_{1}\left(\bar{K}_{t}, H_{t}\right)
$$

where $F_{i}$ denotes the partial derivative of the production function $F$ with respect to $i$ th argument. 
Krusell, Kuruscu and Smith(2002) have emphasized that one needs to allow for the difference between the capital holdings of an individual agent, denoted by $K_{t}$, and the average capital holdings in the economy $\bar{K}_{t}$, in order to analyze a competitive equilibrium in a growth model with quasi-geometric discounting. In particular, we assume that the agent's decision rule for capital holdings can be written as $K_{t+1}=g\left(K_{t}, \bar{K}_{t}, A_{t}\right)$, while the law of motion for the aggregate capital stock is $\bar{K}_{t+1}=G\left(\bar{K}_{t}, A_{t}\right)$, for $t=0,1,2, \cdots, \infty$. A symmetric equilibrium then requires setting $K_{t}=\bar{K}_{t}$, which in turn leads to

$$
g\left(\bar{K}_{t}, \bar{K}_{t}, A_{t}\right)=G\left(\bar{K}_{t}, A_{t}\right)
$$

for $t=0,1, \cdots, \infty$.

We now summarize a set of competitive equilibrium conditions in a stochastic growth model with quasi-geometric discounting. First, the generalized Euler equation (2.6) can be now rewritten in terms of the decision rule for the individual agent's capital holdings:

$$
1=\beta E_{t}\left[\frac{u_{1}\left(C_{t+1}, 1-H_{t+1}\right)}{u_{1}\left(C_{t}, 1-H_{t}\right)}\left[\theta R_{t+1}+(1-\theta) g_{1}\left(\bar{K}_{t+1}, \bar{K}_{t+1}, A_{t+1}\right)\right]\right],
$$

where $R_{t}$ is given by

$$
R_{t}=1-\delta+A_{t} F_{1}\left(\bar{K}_{t}, H_{t}\right)
$$

Also, substituting (2.9) into the utility maximization condition for labor supply (2.5) leads to

$$
\frac{u_{2}\left(C_{t}, 1-H_{t}\right)}{u_{1}\left(C_{t}, 1-H_{t}\right)}=A_{t} F_{2}\left(\bar{K}_{t}, H_{t}\right)
$$

The social resource constraint is given by

$$
C_{t}+\bar{K}_{t+1}=A_{t} F\left(\bar{K}_{t}, H_{t}\right)+(1-\delta) \bar{K}_{t}
$$

In sum, one can compute a sequence of equilibrium quantities $\left\{C_{t}, H_{t}, \bar{K}_{t+1}\right\}_{t=0}^{\infty}$ and a sequence of equilibrium real prices $\left\{W_{t}, R_{t}\right\}_{t=0}^{\infty}$ by solving a system of (2.9), (2.11), (2.12), (2.13), and (2.14) in each period $t=0,1, \cdots, \infty$, given $K_{0}$ and $\left\{A_{t}\right\}_{t=0}^{\infty}$.

\subsection{A Closed Form Solution for the Competitive Equilibrium}

We now consider a closed form solution for the competitive equilibrium. We therefore restrict preference and technology to specific functional forms. We also assume that capital 
depreciates completely within a period, so that we set $\delta=1$.

In what follows, it will be assumed that the aggregate production function takes a Cobb-Douglas form:

$$
Y_{t}=A_{t} \bar{K}_{t}^{\alpha} H_{t}^{1-\alpha}
$$

where $\alpha$ denotes the output elasticity of capital. In addition, the period utility function of the household is given by

$$
u\left(C_{t}, 1-H_{t}\right)=\log C_{t}+b \log \left(1-H_{t}\right)
$$

where $b$ is a positive constant.

Krusell, Kuruscu, and $\operatorname{Smith}(2002)$ show that an individual household has a saving function in a growth model with quasi-geometric discounting, which is linear in his or her capital holdings. We therefore assume that the policy function for an individual household's capital holdings is assumed to be of the form:

$$
g\left(K_{t}, \bar{K}_{t}, A_{t}\right)=\frac{K_{t}}{\bar{K}_{t}} G\left(\bar{K}_{t}, A_{t}\right) .
$$

We also assume that the equilibrium division of output between consumption and investment is assumed to be

$$
\bar{K}_{t+1}=s \alpha Y_{t} ; \quad C_{t}=(1-s \alpha) Y_{t}
$$

where $s$ is a positive constant which will be determined.

It is important to note that $K_{t}=\bar{K}_{t}$ in an equilibrium. It thus follows from $(2.17)$ that the partial derivative of the policy function for the individual household with respect to his or her capital stock can be written as

$$
g_{1}\left(\bar{K}_{t+1}, \bar{K}_{t+1}, A_{t+1}\right)=\frac{\bar{K}_{t+2}}{\bar{K}_{t+1}} .
$$

Hence, given functional forms of utility and production functions described in (2.15) and (2.16), the Euler equation specified in (2.6) can be rewritten as

$$
\frac{\bar{K}_{t+1}}{C_{t}}=\theta \alpha \beta+\beta E_{t}\left[(\alpha \theta+1-\theta) \frac{\bar{K}_{t+2}}{C_{t+1}}\right] .
$$

In addition, note that equation (2.18) leads to $\frac{\bar{K}_{t+1}}{C_{t}}=\frac{s \alpha}{1-s \alpha}$. We, therefore, set $\frac{\bar{K}_{t+1}}{C_{t}}=$ $\frac{s \alpha}{1-s \alpha}$ in $(2.20)$ and then solve the resulting equation for $s$, which in turn leads to

$$
s=\frac{\theta \beta}{1-\beta(1-\theta)} .
$$


Here, one can see from (2.21) that $0<\beta<1$ leads to $0<s<1$, to the extent that $\theta>0$.

Furthermore, we can show that the equilibrium number of hours is constant in each period $t=0,1, \cdots, \infty$. Note that, given functional forms of utility and production functions described in (2.15) and (2.16), the equilibrium condition for labor (2.13) can be rewritten as

$$
\frac{b C_{t}}{1-H_{t}}=(1-\alpha) \frac{Y_{t}}{H_{t}}
$$

Then, setting $\frac{C_{t}}{Y_{t}}=1-s \alpha$ in $(2.22)$, we can see that the equilibrium number of hours is a constant satisfying

$$
\frac{b H}{1-H}=\frac{(1-\alpha)}{1-s \alpha} .
$$

Hence, substituting (2.21) into (2.18), the policy function for the average capital holdings in the economy can be written as

$$
\bar{K}_{t+1}=\frac{\alpha \theta \beta}{1-\beta(1-\theta)} A_{t} \bar{K}_{t}^{\alpha} H^{1-\alpha} .
$$

As a result, one can use equations (2.15), (2.18), and (2.24) to compute a closed form solution for the competitive equilibrium allocation $\left\{C_{t}, Y_{t}, \bar{K}_{t+1}\right\}_{t=0}^{\infty}$ given $\bar{K}_{0}$ and $\left\{A_{t}\right.$ \}$_{t=0}^{\infty}$.

\subsection{The Generalized Euler Equation in the Planner's Problem}

Having described a closed form solution for the competitive equilibrium, we turn to the discussion of the generalized Euler equation for the social planner's problem. The reason why we are concerned with the social planner's problem is that the marginal benefit from a marginal change in the planner's saving differs from that of a competitive household's saving.

Specifically, the social planner's problem can be written as

$$
\max \left\{u\left(C_{t}, 1-H_{t}\right)+\theta \beta\left[\sum_{k=0}^{\infty} \beta^{k} E_{t}\left[u\left(C_{t+k}, 1-H_{t+k}\right)\right]\right]\right\},
$$

subject to

$$
C_{t}+\bar{K}_{t+1}-(1-\delta) \bar{K}_{t} \leq A_{t} F\left(\bar{K}_{t}, H_{t}\right)
$$


for $t=0,1,2, \cdots, \infty$ and given $\bar{K}_{0}$ and $\left\{A_{t}\right\}_{t=0}^{\infty}$. The generalized Euler equation for the social planner's problem is then given by

$$
1=\beta E_{t}\left[\frac{u_{1}\left(C_{t+1}, 1-H_{t+1}\right)}{u_{1}\left(C_{t}, 1-H_{t}\right)}\left[\theta\left(1-\delta+A_{t} F_{1}\left(\bar{K}_{t+1}, H_{t+1}\right)\right)+(1-\theta) G_{1}\left(\bar{K}_{t+1}, A_{t+1}\right)\right]\right] .
$$

In order to obtain a closed form solution for the social planner's problem, we now continue to restrict preference and technology to the same functional forms analyzed in the previous section. We also continue to assume that capital depreciates completely within a period, so that we set $\delta=1$. The generalized Euler equation for the social planner's problem can be therefore rewritten as

$$
\frac{\bar{K}_{t+1}}{C_{t}}=\theta \alpha \beta+\beta E_{t}\left[\left(\alpha \theta+(1-\theta) \epsilon_{k}\left(\bar{K}_{t+1}, A_{t+1}\right)\right) \frac{\bar{K}_{t+2}}{C_{t+1}}\right],
$$

where $\epsilon_{k}\left(\bar{K}_{t+1}, A_{t+1}\right)$ denotes the elasticity of the next period's capital stock with respect to the current period's capital stock:

$$
\epsilon_{k}\left(\bar{K}_{t+1}, A_{t+1}\right)=\frac{\partial \bar{K}_{t+2}}{\partial \bar{K}_{t+1}} \frac{\bar{K}_{t+1}}{\bar{K}_{t+2}} .
$$

We then use a 'guess and verify method' to compute a closed form solution for the model. Specifically, suppose that the elasticity of the next period's capital stock with respect to the current period's capital stock is constant over time, so that $\epsilon_{k}\left(\bar{K}_{t+1}, A_{t+1}\right)$ $=\epsilon_{k}$ in each period $t=0,1, \cdots, \infty$. A successive forward iteration of (2.28) then leads to

$$
\bar{K}_{t+1}=\frac{\alpha \beta \theta}{1-\beta\left(\alpha \theta+(1-\theta) \epsilon_{k}\right)} C_{t},
$$

where the following terminal condition is satisfied:

$$
\lim _{T \rightarrow \infty}\left(\beta\left(\alpha \theta+(1-\theta) \epsilon_{k}\right)\right)^{T} E_{t}\left[\frac{\bar{K}_{t+T+1}}{C_{t+T}}\right]=0 .
$$

Substituting $C_{t}=Y_{t}-\bar{K}_{t+1}$ into (2.30) and rearranging, we have

$$
\bar{K}_{t+1}=\frac{\alpha \beta \theta}{1-\beta(1-\theta) \epsilon_{k}} Y_{t} .
$$

Notice that firms produce output using a Cobb-Douglas production function. Hence, one can see from $(2.29)$ and $(2.31)$ that $\epsilon_{k}=\alpha$. In addition, the number of hours worked is constant for the planner's problem if one assumes logarithmic utility function, CobbDouglas production function and complete depreciation of capital. Hence, substituting 
(2.30) into (2.26) and then setting $\epsilon_{k}=\alpha$ in the resulting equation, we can see that the capital stock at the next period therefore can be written as

$$
\bar{K}_{t+1}=\frac{\theta \alpha \beta}{1-\alpha \beta(1-\theta)} A_{t} \bar{K}_{t}^{\alpha} \bar{H}^{1-\alpha},
$$

where $\bar{H}$ is defined as

$$
\bar{H}=\frac{(1-\alpha)(1-\alpha \beta(1-\theta))}{b(1-\alpha \beta)+(1-\alpha)(1-\alpha \beta(1-\theta))} .
$$

As a result, one can use equations (2.30), (2.31), and (2.32) to compute a closed form solution for the planner's problem $\left\{C_{t}, Y_{t}, \bar{K}_{t+1}\right\}_{t=0}^{\infty}$ given $\bar{K}_{0}$ and $\left\{A_{t}\right\}_{t=0}^{\infty}$.

Next, we turn to the discussion of the relationship between observable and unobservable terms in the generalized Euler equation. It is here important to note that quasi-geometric discounting generates an unobservable term in the generalized Euler equation, which is absent in the case of ordinary geometric discounting. More explicitly, the unobservable term for the planner's problem corresponds to $G_{1}\left(\bar{K}_{t}, A_{t}\right)$, while competitive equilibrium creates $g_{1}\left(\bar{K}_{t}, \bar{K}_{t}, A_{t}\right)$. We then show that the complete depreciation of capital makes the unobservable term proportional to the observable return for both of the competitive equilibrium and the planner's problem, given the functional forms of preference and technology (2.15) and (2.16).

First, we consider the unobservable term in the planner's problem. We then show that the partial derivative of the decision rule for the average capital holdings is proportional to the marginal product of capital, which is denoted by $R_{t}^{*}$. Specifically, we can see from (2.17) and (2.32) that the observable term in the generalized Euler equation is given by

$$
G_{1}\left(\bar{K}_{t}, A_{t}\right)=s^{*} R_{t}^{*}
$$

where $s^{*}$ is defined as

$$
s^{*}=\frac{\theta \alpha \beta}{1-\alpha \beta(1-\theta)} .
$$

We now turn to the case of the competitive equilibrium. In the case of the competitive equilibrium, one can use equations (2.17) and (2.24) to obtain the following relationship between observable and unobservable returns:

$$
g_{1}\left(\bar{K}_{t}, \bar{K}_{t}, A_{t}\right)=\frac{G\left(\bar{K}_{t}, A_{t}\right)}{\bar{K}_{t}}=s R_{t},
$$


where $s$ is given by

$$
s=\frac{\theta \beta}{1-\beta(1-\theta)} .
$$

We can see from generalized Euler equations for the competitive household and the so-

cial planner (2.11) and (2.27) that when unobservable terms are considered as parts of investment returns, the investment return includes observable and unobservable terms as its components. It also follows from (2.35) and (2.37) that proportionality constants $s$ and $s^{*}$ are less than 1 if $0<\alpha<1,0<\beta<1$ and $0<\theta<1$. Hence, households may have a smaller size of investment return under quasi-geometric discounting than under ordinary geometric discounting. In the next section, therefore, we will discuss implications of quasi-geometric discounting on the Sharpe ratio of investment return.

\section{Implications of Quasi-geometric Discounting for Asset Pricing}

Having derived the Euler equation (2.11), we now show that time-inconsistent preferences can help modify the standard bound on the coefficient of variation of the ratio of marginal utilities of consumption across periods.

\subsection{The Relationship between the Sharpe Ratio and the Stochastic Discount Factor under Quasi-geometric Discounting}

In this section, we discuss the relationship between the Sharpe ratio of investment and the stochastic discount factor under quasi-geometric discounting. In doing so, we emphasize that generalized Euler equations for the competitive household and the social planner (2.11) and (2.27) include unobservable terms respectively. The presence of such unobservable terms is a product of strategic interaction between the consumer's current and future selves, given time inconsistent preferences. We also consider the unobservable terms as parts of investment returns. Hence, investment under quasi-geometric discounting creates observable and unobservable returns.

We then show that a candidate for the stochastic discount factor in a stochastic growth model with quasi-geometric discounting is the intertemporal marginal rate of substitution of a consumer in models with ordinary geometric discounting. Specifically, let $M_{t+1}$ denote the intertemporal marginal rate of substitution between consumptions at periods $t$ 
and $t+1$ of consumers that behave as if they discount future utilities at a constant rate:

$$
M_{t+1}=\beta \frac{u_{1}\left(C_{t+1}, 1-H_{t+1}\right)}{u_{1}\left(C_{t}, 1-H_{t}\right)} .
$$

Consider the generalized Euler equation for a competitive household. It will be shown that given the definition of the stochastic discount factor (3.1), one can use the Euler equation (2.11) to decompose the whole investment return into its observable and unobservable components. In order to see this, let $R_{i, t+1}$ and $X_{i, t+1}$, respectively, denote the observable and unobservable investment returns at period $t+1$, which are defined as

$$
R_{i, t+1}=1-\delta+\hat{R}_{t+1} ; \quad X_{i, t+1}=g_{1}\left(\bar{K}_{t+1}, \bar{K}_{t+1}, A_{t+1}\right) .
$$

The whole investment return at period $t+1$ is then defined as a weighted average of its observable and unobservable components:

$$
\tilde{R}_{i, t+1}=\theta R_{i, t+1}+(1-\theta) X_{i, t+1}
$$

where $\tilde{R}_{i, t+1}$ denotes the whole investment return at period $t+1$ that consumers expect to obtain when they hold it for one period. The weight for the observable return is the measure of the short-run impatience, $\theta$. Substituting (3.1) and (3.3) into (2.11), the generalized Euler equation can be rewritten as

$$
1=E_{t}\left[M_{t+1} \tilde{R}_{i, t+1}\right]
$$

It is well-known in the literature on asset pricing that the expected value of the stochastic discount factor times the return of an asset is equal to one in a consumption-based asset pricing model. Given definitions of the discount factor $M_{t+1}$, and the observable and unobservable investment returns $R_{i, t+1}$ and $\tilde{R}_{i, t+1}$, we can see from equation (3.4) that the generalized Euler equation leads to such a basic formula for asset pricing.

In the case of the planner's problem, define $R_{i, t+1}$ and $X_{i, t+1}$ as

$$
R_{i, t+1}=R_{t+1}^{*} ; \quad X_{i, t+1}=G_{1}\left(\bar{K}_{t+1}, A_{t+1}\right),
$$

where $R_{t+1}^{*}$ denotes the observable return for the planner's problem, defined by

$$
R_{t+1}^{*}=1-\delta+A_{t} F_{1}\left(\bar{K}_{t+1}, H_{t+1}\right)
$$


Then, one can see that the generalized Euler equation (2.27) leads to the basic formula for asset pricing (3.4).

We now turn to the discussion of an expected risk-premium on the investment return. In order to do so, we assume that there is a portfolio whose return has a zero covariance with the stochastic discount factor. Specifically, let $R_{z, t+1}$ denote the return at period $t+1$ of the zero covariance portfolio. It then follows from (3.4) that the relationship between the conditional expectation of the stochastic discount factor and the expected return of the zero covariance portfolio can be written as

$$
1=E_{t}\left[M_{t+1}\right] E_{t}\left[R_{z, t+1}\right]
$$

Then, following the literature on asset pricing, equations (3.4) and (3.7) can be solved to show that the expected excess return on any asset $i$ satisfies

$$
E_{t}\left[\tilde{R}_{i, t+1}\right]-E_{t}\left[R_{z, t+1}\right]=-\frac{\operatorname{cov}_{t}\left(M_{t+1}, \tilde{R}_{i, t+1}\right)}{E_{t}\left[M_{t+1}\right]}
$$

where $\operatorname{cov}_{t}(x, y)$ denotes the conditional covariance of random variables $x$ and $y$ conditional on the information set at period $t$. The characterization of the expected excess return described in (3.8) indicates that the expected excess return of investment is determined by its risk, as measured by the negative covariance with stochastic discount factor divided by the expected stochastic discount factor.

Because the absolute value of the correlation between the stochastic discount factor and an asset's return must be less than one, the negative covariance in equation (3.8) must be less than the product of the standard deviations of the asset's return and the stochastic discount factor. It thus implies that we have

$$
\frac{E_{t}\left[\tilde{R}_{i, t+1}\right]-E_{t}\left[R_{z, t+1}\right]}{\sigma_{t}\left(\tilde{R}_{i, t+1}\right)} \leq \frac{\sigma_{t}\left(M_{t+1}\right)}{E_{t}\left[M_{t+1}\right]},
$$

where $\sigma_{t}(x)$ denotes the conditional standard deviation of a variable $x$. The ratio of the expected excess investment return to its standard deviation puts a lower bound on the ratio of the second moment to the first moment of the stochastic discount factor, as analyzed in the works of Shiller(1982) and Hansen and Jaganathan(1991). 


\subsection{The Observable and Unobservable Sharpe Ratios under Quasi-geometric Discounting}

It is important to note that the whole investment return is unobservable. It means that equation (3.9) itself is not useful to see whether time-inconsistent preferences help modify the bound on the coefficient of variation on the stochastic discount factor. To get around such a problem, one can rewrite (3.9) in terms of the observable Sharpe ratio. Specifically, let $\tilde{S} R_{i, t}$ denote the Sharpe ratio of the asset $i$ in terms of its whole return, which is defined as the asset's risk premium divided by its return's standard deviation:

$$
\tilde{S} R_{i, t}=\frac{E_{t}\left[\tilde{R}_{i, t+1}\right]-E_{t}\left[R_{z, t+1}\right]}{\sigma_{t}\left(\tilde{R}_{i, t+1}\right)} .
$$

The observable Sharpe ratio of the asset is then defined in terms of its observable return:

$$
S R_{i, t}=\frac{E_{t}\left[R_{i, t+1}\right]-E_{t}\left[R_{z, t+1}\right]}{\sigma_{t}\left(R_{i, t+1}\right)} .
$$

Substituting (3.3) into (3.10) and rearranging, we have

$$
\tilde{S} R_{i, t}=\frac{\sigma_{t}\left(R_{i, t+1}\right)}{\sigma_{t}\left(\tilde{R}_{i, t+1}\right)} S R_{i, t}-(1-\theta) \frac{E_{t}\left[R_{i, t+1}\right]-E_{t}\left[X_{i, t+1}\right]}{\sigma_{t}\left(\tilde{R}_{i, t+1}\right)} .
$$

Thus, we can see that the unobservable Sharpe ratio has a linear relationship with the observable Sharpe ratio.

In order to get insights about the role of quasi-geometric discounting, we now turn to the discussion of a closed form relationship between unobservable and observable Sharpe ratios, which is implied by closed form solutions for the competitive equilibrium and the

planner's problem. Given the logarithmic utility function for consumption specified in (2.16), it follows from (3.1) that the stochastic discount factor is given by

$$
M_{t+1}=\beta \frac{C_{t}}{C_{t+1}} .
$$

It also follows from (2.34) and (2.36) that the unobservable investment return is proportional to the observable investment return:

$$
X_{i, t+1}=\phi R_{i, t+1}
$$

where $\phi$ is a positive constant. More explicitly, $\phi=s$ for the competitive equilibrium and $\phi=s^{*}$ for the planner's problem. The whole investment return then becomes proportional 
to the observable return:

$$
\tilde{R}_{i, t+1}=(\theta+(1-\theta) \phi) R_{i, t+1} .
$$

Substituting (3.14) and (3.15) into (3.12), the whole Sharpe ratio of the asset $i$ can be written as

$$
\tilde{S} R_{i, t}=\frac{1}{\theta+(1-\theta) \phi}\left(S R_{i, t}-(1-\theta)(1-\phi) \frac{E_{t}\left[R_{i, t+1}\right]}{\sigma_{t}\left(R_{i, t+1}\right)}\right) .
$$

Next, we turn to the discussion of sufficient conditions for $\tilde{S} R_{i, t}<S R_{i, t}$. In order to do this, we compute the difference between the unobservable and observable Sharpe ratios by subtracting $S R_{i, t}$ from both sides of (3.16):

$$
\tilde{S} R_{i, t}-S R_{i, t}=\frac{(1-\theta)(1-\phi)}{\theta+(1-\theta) \phi}\left(S R_{i, t}-\frac{E_{t}\left[R_{i, t+1}\right]}{\sigma_{t}\left(R_{i, t+1}\right)}\right)
$$

Then, substituting (3.11) into the right-hand side of (3.17) and setting $E_{t}\left[R_{z, t+1}\right]=\frac{1}{E_{t}\left[M_{t+1}\right]}$ in the resulting equation, we have

$$
\tilde{S} R_{i, t}-S R_{i, t}=-\left\{\frac{(1-\theta)(1-\phi)}{\theta+(1-\theta) \phi}\right\} \frac{1}{\sigma_{t}\left(R_{i, t+1}\right) E_{t}\left[M_{t+1}\right]} .
$$

Therefore, to the extent that $\sigma_{t}\left(R_{i, t+1}\right)>0$ and $E_{t}\left[M_{t+1}\right]>0$, equation (3.18) implies that sufficient conditions for $\tilde{S} R_{i, t}<S R_{i, t}$ can be written as

$$
0<\theta<1 ; \quad 0<\phi<1
$$

It is now important to note that under a quasi-geometric discounting, a lower bound of the coefficient of variation on the stochastic discount factor is $\tilde{S} R_{i, t}$, while the lower bound under ordinary geometric discounting corresponds to $S R_{i, t}$. Therefore, the sufficient conditions for $\tilde{S} R_{i, t}<S R_{i, t}$ described in (3.19) raise the possibility that quasi-geometric discounting can help modify the standard lower bound on the coefficient of variation of the stochastic discount factor.

In order to see this, note that the investment return under quasi-geometric discounting is a weighted average of unobservable and observable terms, with the weight for the observable term $\theta$. It thus implies that if the unobservable investment return is less than the observable investment return, the whole investment return should remain below the observable return. Specifically, $0<\phi<1$ guarantees that the unobservable term in the generalized Euler equation is less than the observable term. In addition, it follows from 
(2.35) and (2.37) that $\phi=s$ for the competitive equilibrium and $\phi=s^{*}$ for the planner's problem. We also know that both $s$ and $s^{*}$ are less than 1 , given the closed form solutions described above. It thus follows from (3.15) that a rise in the short-run impatience of households decreases the whole investment return, given a level of the observable return. This in turn implies that the absolute value of the conditional covariance between the stochastic discount factor and the whole investment return can take a smaller value under quasi-geometric discounting than under ordinary geometric discounting for a range of values of the short-run impatience of households. Hence, to the extent that the closed form solutions hold, we find that when discount rates of households are greater in the shortrun than in the long-run and capital depreciates completely within a period, investment creates a smaller size of the expected risk premium under quasi-geometric discounting than under ordinary geometric discounting. Therefore, the unobservable Sharpe ratio for the investment under quasi-geometric discounting remains below the observable Sharpe ratio. As a result, we can conclude that the lower bound of the coefficient variation of the stochastic discount factor is smaller under quasi-geometric discounting than under ordinary geometric discounting, if the conditions specified in (3.19) are satisfied.

\subsection{The Computation of the Unobservable Sharpe Ratio}

Having described the relationship between the unobservable and observable Sharpe ratios, we will compute the unobservable Sharpe ratio on the basis of the observable Sharpe ratio. In particular, we will assume that technology shocks follow a normal distribution. This in turn implies that the observable return follow a log-normal distribution, so long as the closed form solutions analyzed in the previous section hold. It also follows from the log-normality of the observable return that the ratio of the conditional expectation of the observable return to its conditional standard deviation can be written as

$$
\frac{E_{t}\left[R_{i, t+1}\right]}{\sigma_{t}\left(R_{i, t+1}\right)}=\left(\exp \left(\sigma_{t}\left(r_{i, t+1}\right)^{2}\right)-1\right)^{-\frac{1}{2}}
$$

where $\sigma_{t}\left(R_{i, t+1}\right)$ denotes the conditional standard deviation of the observable return and $r_{i, t+1}=\log R_{i, t+1}$. Therefore, substituting (3.20) into (3.16), we have

$$
\tilde{S} R_{i, t}=\frac{S R_{i, t}}{\theta+(1-\theta) \phi}-\frac{(1-\theta)(1-\phi)}{\theta+(1-\theta) \phi}\left(\exp \left(\sigma_{t}\left(r_{i, t+1}\right)^{2}\right)-1\right)^{-\frac{1}{2}} .
$$


Note that in the case of complete depreciation of capital, the observable return equals the marginal product of capital. Thus, given that we have the closed form solutions for the competitive equilibrium and the planner's problem described above, the logarithm of the observable return can be written as

$$
r_{i, t}=\log \alpha+(1-\alpha) \log \bar{H}+a_{t}-(1-\alpha) \log \bar{K}_{t}
$$

The conditional standard deviation of the logarithm of the observable return therefore is given by

$$
\sigma_{t}\left(r_{i, t+1}\right)^{2}=\sigma_{a}^{2}
$$

As a result, substituting (3.23) into (3.21), we have a closed form relationship between the unobservable and observable investment returns:

$$
\tilde{S} R_{i, t}=\frac{S R_{i, t}}{\theta+(1-\theta) \phi}-\frac{(1-\theta)(1-\phi)}{\theta+(1-\theta) \phi}\left(\exp \left(\sigma_{a}^{2}\right)-1\right)^{-\frac{1}{2}} .
$$

Having discussed the unobservable Sharpe ratio, we now turn to the discussion of how to compute the ratio of the second moment of the stochastic discount factor to its first moment. First, under the assumption of the log-normality, we can write the coefficient of variation of the stochastic discount factor as follows:

$$
\frac{\sigma_{t}\left(M_{t+1}\right)}{E_{t}\left[M_{t+1}\right]}=\left(\exp \left(\sigma_{t}\left(m_{t+1}\right)^{2}\right)-1\right)^{\frac{1}{2}}
$$

where $m_{t+1}=\log M_{t+1}$. It also follows from the logarithmic utility function for consumption that the conditional standard deviation of the logarithm of the stochastic discount factor equals that of the logarithm of consumption growth for both the competitive equilibrium and the planner's problem. We also use equations (2.18) and (2.24) for the competitive equilibrium and equations (2.30) and (2.32) for the planner's problem respectively, in order to show that the conditional standard deviation of the logarithm of consumption growth equals that of the technology shock. As a result, the coefficient of variation of the stochastic discount factor can be written as

$$
\frac{\sigma_{t}\left(M_{t+1}\right)}{E_{t}\left[M_{t+1}\right]}=\left(\exp \left(\sigma_{a}^{2}\right)-1\right)^{\frac{1}{2}}
$$

As a result, given that equation (3.24) for the unobservable Sharpe ratio holds, the relationship between the unobservable Sharpe ratio and the coefficient of variation of the 
stochastic discount factor specified in equation (3.9) can be rewritten as

$$
\tilde{S} R_{i, t} \leq\left(\exp \left(\sigma_{a}^{2}\right)-1\right)^{\frac{1}{2}}
$$

Having written the relationship between the unobservable and observable Sharpe ratios, we will compute the relationship between the unobservable Sharpe ratio and the short-run impatience of households, given observed values of the observable Sharpe ratio. We do this, in order to see if quasi-geometric discounting can generate a significant change in the lower bound of the stochastic discount factor. We therefore need to calibrate values for parameters $\alpha, \beta, \theta, \sigma_{a}, \rho_{a}$. Specifically, the steady state Euler equation is used to set a value of the long-run discount factor:

$$
\beta=\frac{1}{(\theta+(1-\theta) \phi) R}
$$

where $R$ denotes the long-run average value of the observable return. Notice from (2.35) and (2.37) that $\phi=s$ for the competitive equilibrium and $\phi=s^{*}$ for the planner's problem. Therefore, values of long-run time discount factor for the competitive equilibrium and the planner's problem, respectively, are given by

$$
\beta=\frac{1}{\theta R+1-\theta} ; \quad \beta^{*}=\frac{1}{\theta R+\alpha(1-\theta)},
$$

where $\beta^{*}$ denotes the long-run time discount factor for the planner's problem. The persistence parameter of the logarithm of the total factor productivity is set to be $\rho_{a}=0.95$ and the standard deviation of technology shock is $\sigma_{a}=0.00785$, which are taken from Hansen(1985). In addition, the labor income share is given by $s_{H}=0.58$, which corresponds to setting $\alpha=0.42$, following King, Plosser, and Rebelo (1988a, b). The parameter values used in this paper are reported in Table 1.

Table 2 reports annual average values of returns on stocks and bonds and their standard deviations over the sample period from 1947 through 1996, which are taken from Cochrane(1997). For example, the annual average return from the value weighted NYSE portfolio is $9.1 \%$, which corresponds to $R=1.0225$. The standard deviation of the real return on the value weighted NYSE portfolio is $16.7 \%$. In addition, when the Sharpe ratio is defined as the ratio of the average excess return of stocks to the standard deviation of stock return, it is 0.497 for the value weighted NYSE portfolio, 0.561 for S\&P 500, 
and 0.459 for the equally weighted NYSE portfolio. Table 2 therefore indicates that the Sharpe ratios for the three measures of real returns on stocks and bonds are near 0.5 in the postwar U.S. data.

Figures 1 and 2 report the relationship between the short-run impatience of households and the unobservable Sharpe ratio for the competitive equilibrium and the planner's problem respectively, while the short-run impatience is represented by $\theta$. The value of the observable Sharpe ratio is set equal to the Sharpe ratio of the value weighted NYSE portfolio, the equally weighted NYSE portfolio and S\&P 500 respectively. Figures 1 and 2 demonstrate that as the short-run impatience of households increases, the unobservable Sharpe ratio falls.

Comparing Figure 1 with Figure 2, one can see that the unobservable Sharpe ratio for the competitive equilibrium remains above that of the planner's problem. Specifically, the unobservable Sharpe ratio for the competitive equilibrium can match the coefficient of variation of the stochastic discount factor at $\theta=0.827,0.805$, and 0.841 for the equally weighted NYSE, S\&P 500, and the value weighted NYSE respectively, given parameter values described above. However, the planner's problem requires $\theta=0.994,0.993$, and 0.994, for the equally weighted NYSE, S\&P 500, and the value weighted NYSE respectively, in order to match the coefficient of variation of the stochastic discount factor.

The reason why the competitive equilibrium generates a higher level of the unobservable Sharpe ratio than the planner's problem can be explained as follows. It follows from decision rules for capital holdings specified in (2.17) and (2.32) that the planner's decision rule is decreasing in capital holdings, while the competitive equilibrium consumer's decision rule is linear in his or her capital holdings. Therefore, when discount rates of households are greater in the short-run than in the long-run, the competitive equilibrium consumer sees a higher benefit from extra saving today than does the planner. Therefore, everything else equal, the planner sees another unit of savings as yielding a smaller increase in future savings than does the competitive equilibrium consumer, as discussed in Krusell, Kuruscu, and Smith(2002). This in turn implies that when the observable return is taken as given, the unobservable return from investment is smaller for the planner's problem than for the competitive equilibrium. Since the whole investment return is a weighted average of observable and unobservable terms, it means that the whole investment return is 
greater for the competitive equilibrium than for the planner's problem. The unobservable Sharpe ratio is thus higher for the competitive equilibrium than for the planner's problem. As a result, the competitive equilibrium requires a higher level of short-run impatience of households in order to make the unobservable Sharpe ratio equal the coefficient of variation of the stochastic discount factor.

\subsection{The Relationship between the Observable Sharpe Ratio and the Stochas- tic Discount Factor in the Presence of State Contingent Claims}

In this section, we consider an example in which quasi-geometric discounting may not help to modify the standard lower bound on the coefficient of variation of the stochastic discount factor, unless the unobservable return from investment analyzed in the previous section is taken into account. The sole difference from the previous section is the presence of one-period ahead state contingent claims in sequentially complete markets, while the previous section assumes that state contingent claims are not traded in markets. We then derive the relationship between the observable Sharpe ratio and the stochastic discount factor in the presence of state contingent claims on consumption goods in sequentially complete markets. In particular, we focus on a closed-form solution for the competitive equilibrium in the presence of state contingent claims. Hence, we continue to restrict preference and technology to the same functional forms employed in the previous section.

The preference at period $t$ of the representative household is represented by the following utility function:

$$
\log \left(C_{t}\right)+b \log \left(1-H_{t}\right)+\theta \beta\left[\sum_{k=0}^{\infty} \beta^{k} E_{t}\left[\log \left(C_{t+1+k}\right)+b \log \left(1-H_{t+1+k}\right)\right]\right] .
$$

The period budget constraint at period $t$ of the representative household is also given by

$$
C_{t}+E_{t}\left[Q_{t, t+1} B_{t+1}\right]+K_{t+1} \leq B_{t}+W_{t} H_{t}+R_{t} K_{t}
$$

where $Q_{t, t+1}$ denotes the stochastic discount factor for computing the price at period $t$ of one unit of consumption goods at period $t+1$. Here, $B_{t+1}$ denotes one-period ahead state contingent bond, which delivers one unit of consumption goods at period $t+1$ only if a specific state occurs at period $t+1$ and gives nothing otherwise. Let $N_{t}$ denote the real 
wealth of the representative household, which is defined by

$$
N_{t}=B_{t}+R_{t} K_{t}
$$

In addition, no-arbitrage condition implies that the following equality holds:

$$
1=E_{t}\left[Q_{t, t+1} R_{t+1}\right]
$$

Substituting (3.32) and (3.33) into (3.31), we can see that the period budget constraint of the consumer can be rewritten as

$$
C_{t}+E_{t}\left[Q_{t, t+1} N_{t+1}\right] \leq W_{t} H_{t}+N_{t}
$$

Having described the period budget constraint of the household in terms of his or her real wealth, we now turn to the discussion of the behavior of the household. In order to do this, let $V\left(N_{t}, \bar{N}_{t}, A_{t}\right)$ be the value function at period $t$, which satisfies

$$
V\left(N_{t}, \bar{N}_{t}, A_{t}\right)=\left\{\log C_{t}+b \log \left(1-H_{t}\right)+\beta E_{t}\left[V\left(N_{t+1}, \bar{N}_{t+1}, A_{t+1}\right)\right]\right\},
$$

subject to the constraint (3.34). Here, $\bar{N}_{t}$ denotes the average real wealth at period $t$ in the economy. The optimization of the current self at period $t$ of the representative household then can be written as

$$
F\left(N_{t}, \bar{N}_{t}, A_{t}\right)=\max _{\left\{C_{t}, N_{t+1}\right\}}\left\{\log C_{t}+b \log \left(1-H_{t}\right)+\theta \beta E_{t}\left[V\left(N_{t+1}, \bar{N}_{t+1}, A_{t+1}\right)\right]\right\},
$$

subject to the period budget constraint (3.34). Here, $F\left(N_{t}, \bar{N}_{t}, A_{t}\right)$ denotes the value function of the current self at period $t$. The first-order condition for $N_{t+1}$ is given by

$$
Q_{t, t+1}\left(\frac{1}{C_{t}}\right)=\theta \beta V_{1}\left(N_{t+1}, \bar{N}_{t+1}, A_{t+1}\right),
$$

which holds at every state in period $t+1$. The first-order condition for labor supply is

$$
W_{t}=b \frac{C_{t}}{1-H_{t}}
$$

The envelope condition for (3.36) is

$$
F_{1}\left(N_{t}, \bar{N}_{t}, A_{t}\right)=\frac{1}{C_{t}}
$$


Combining (3.35) with (3.36), we have

$$
\theta V\left(N_{t}, \bar{N}_{t}, A_{t}\right)=F\left(N_{t}, \bar{N}_{t}, A_{t}\right)-(1-\theta)\left(\log C_{t}+b \log \left(1-H_{t}\right)\right)
$$

Differentiating (3.40) then leads to

$$
\theta V_{1}\left(N_{t}, \bar{N}_{t}, A_{t}\right)=F_{1}\left(N_{t}, \bar{N}_{t}, A_{t}\right)-(1-\theta)\left(\frac{1}{C_{t}}\right)\left(1-E_{t}\left[\frac{\partial N_{t+1}}{\partial N_{t}} Q_{t, t+1}\right]\right) .
$$

Substituting (3.39) into (3.41) also leads to

$$
\theta V_{1}\left(N_{t}, \bar{N}_{t}, A_{t}\right)=\frac{1}{C_{t}}\left(\theta+(1-\theta) E_{t}\left[\frac{\partial N_{t+1}}{\partial N_{t}} Q_{t, t+1}\right]\right)
$$

Combining (3.37) and (3.42), the first-order condition for the current self at period $t$ can be rewritten as

$$
Q_{t, t+1}=\beta \frac{C_{t}}{C_{t+1}}\left(\theta+(1-\theta) E_{t+1}\left[\frac{\partial N_{t+2}}{\partial N_{t+1}} Q_{t+1, t+2}\right]\right) .
$$

In order to characterize the competitive equilibrium in terms of decision rules for real wealth, it is now useful to distinguish between decision rules for the individual consumer's real wealth and the average real wealth in the economy. Specifically, the decision rule for the individual consumer's real wealth is given by

$$
N_{t+1}=x\left(N_{t}, \bar{N}_{t}, A_{t}\right)
$$

while the decision rule for the average real wealth is

$$
\bar{N}_{t+1}=X\left(\bar{N}_{t}, A_{t}\right)
$$

where $\bar{N}_{t}$ denotes the average real wealth in the economy. We assume that the decision rule for the individual consumer's real wealth is given by

$$
x\left(N_{t}, \bar{N}_{t}, A_{t}\right)=\frac{N_{t}}{\bar{N}_{t}} X\left(\bar{N}_{t}, A_{t}\right)
$$

Then, the partial derivative of the decision rule for the individual consumer's real wealth with respect to his or her wealth can be written as

$$
\frac{\partial N_{t+1}}{\partial N_{t}}=\frac{X\left(\bar{N}_{t}, A_{t}\right)}{\bar{N}_{t}} .
$$

Next, we will analyze a closed form solution for the competitive equilibrium. We then use it to compute the stochastic discount factor in the presence of state contingent claims. 
In doing so, we assume that the consumer consumes a constant fraction of his or her wealth at the beginning of each period. Hence, the equilibrium level of consumption is proportional to the average real wealth in the economy:

$$
C_{t}=\omega \bar{N}_{t}
$$

Since all households are the same, $B_{t}=0$ should hold in an equilibrium. In addition, when capital depreciates completely within a period, the real return on investment equals the marginal product of capital:

$$
R_{t}=\alpha \frac{Y_{t}}{K_{t}}
$$

It thus follows from (3.32) and (3.49) that the average real wealth in the economy is given by

$$
\bar{N}_{t}=\alpha Y_{t}
$$

Substituting (3.48) and (3.50) into the social resource constraint, we can see that the next period's capital stock is proportional to the average real wealth in the economy:

$$
K_{t+1}=\frac{1-\alpha \omega}{\alpha} \bar{N}_{t}
$$

The labor income is also given by

$$
W_{t} H_{t}=\frac{1-\alpha}{\alpha} \bar{N}_{t}
$$

The law of motion for the average real wealth in the economy is

$$
\bar{N}_{t+1}=\alpha\left(\frac{1-\alpha \omega}{\alpha}\right)^{\alpha} H^{1-\alpha} A_{t} \bar{N}_{t}^{\alpha},
$$

where $H$ satisfies

$$
H=\frac{1-\alpha}{1-\alpha+\alpha \omega b} .
$$

Substituting (3.48), (3.51), and (3.52) into the period budget constraint, we have

$$
E_{t}\left[Q_{t, t+1} \frac{\bar{N}_{t+1}}{\bar{N}_{t}}\right]=\left(\frac{1}{\alpha}-\omega\right) .
$$

Note that consumption is proportional to the average real wealth in the economy. The first-order condition can be rewritten as

$$
Q_{t, t+1} \frac{\bar{N}_{t+1}}{\bar{N}_{t}}=\beta\left(\theta+(1-\theta) \epsilon E_{t+1}\left[Q_{t+1, t+2} \frac{\bar{N}_{t+2}}{\bar{N}_{t+1}}\right]\right),
$$


where $\epsilon$ denotes the elasticity of the decision rule for the individual consumer's real wealth with respect to his or her real wealth. Taking conditional expectation to both sides of this equation and substituting (3.54) into the resulting equation, we have

$$
\left(\frac{1}{\alpha}-\omega\right)=\beta\left(\theta+(1-\theta) \epsilon\left(\frac{1}{\alpha}-\omega\right)\right) .
$$

Solving this equation for $\omega$ leads to

$$
\omega=\frac{1-\beta(\alpha \theta+(1-\theta) \epsilon)}{\alpha(1-\beta(1-\theta) \epsilon)} .
$$

This in turn implies that

$$
1-\alpha \omega=\frac{\alpha \beta \theta}{1-\beta(1-\theta) \epsilon} .
$$

Substituting this equation into the first-order condition for the current self at period $t$ and rearranging, one can see that one period state contingent price can be written as

$$
Q_{t, t+1}=\left\{\frac{\beta \theta}{1-\beta(1-\theta) \epsilon}\right\} \frac{C_{t}}{C_{t+1}} .
$$

In particular, we can see from (3.46) that $\epsilon=1$ at an equilibrium in which $N_{t}=\bar{N}_{t}$. Hence, when state contingent claims on consumption goods are traded in sequentially complete markets, the stochastic discount factor is given by

$$
\bar{M}_{t+1}=\left\{\frac{\beta \theta}{1-\beta(1-\theta)}\right\} \frac{C_{t}}{C_{t+1}},
$$

where $\bar{M}_{t+1}$ denotes the stochastic discount factor. It then follows from (3.33) and (3.56) that the basic formula for asset pricing is given by

$$
1=E_{t}\left[\bar{M}_{t+1} R_{i, t+1}\right]
$$

were $R_{i, t+1}$ denotes the observable investment return, so that $R_{i, t+1}=R_{t+1}$. Here, we can see from equation (3.57) that the conditional expectation of the stochastic discount factor times the observable return from investment equals one. This is in contrast with equation (3.4), which tells that the conditional expectation of the stochastic discount factor times the unobservable whole return from investment equals one.

We have discussed two alternative representations of the basic formula for asset pricing, both of which are derived from the same competitive equilibrium. It, however, does 
not mean that we have two different sets of competitive equilibrium conditions for the same economy considered in this paper. In particular, we can show that equations (3.4) and (3.57) are the two alternative representations for the same equilibrium condition, depending on whether to allow for the unobservable investment return in a stochastic growth model with quasi-geometric discounting. In order to see this, substituting (3.13) and (3.15) into (3.4) and setting $\phi=s$ in the resulting equation, we have

$$
1=E_{t}\left[\left\{\frac{\beta \theta}{1-\beta(1-\theta)}\right\} \frac{C_{t}}{C_{t+1}} R_{i, t+1}\right],
$$

Given the definition of the stochastic discount factor specified in (3.56), we can see that equation (3.58) is equivalent to equation (3.57). As a result, we can see that equations (3.4) and (3.57) are the two alternative representations for the same equilibrium condition.

We now use (3.57) to obtain an expected risk-premium on the investment return. Specifically, we assume that there is a risk-free asset, which gives one unit of consumption goods, no matter what state occurs at period $t+1$. It then follows from (3.57) that the gross rate of return on the risk-free asset, denoted by $R_{f, t+1}$, can be written as

$$
1=E_{t}\left[\bar{M}_{t+1}\right] R_{f, t+1}
$$

Then, as discussed earlier, we can solve equations (3.57) and (3.59) to show that the relationship between the observable Sharpe ratio and the coefficient of variation of the stochastic discount factor can be written as

$$
\frac{E_{t}\left[R_{i, t+1}\right]-R_{f, t+1}}{\sigma_{t}\left(R_{i, t+1}\right)} \leq \frac{\sigma_{t}\left(\bar{M}_{t+1}\right)}{E_{t}\left[\bar{M}_{t+1}\right]}
$$

Furthermore, given that technology shocks follow a normal distribution, we have

$$
\frac{\sigma_{t}\left[\bar{M}_{t+1}\right]}{E_{t}\left(\bar{M}_{t+1}\right)}=\left(\exp \left(\sigma_{t}\left(\bar{m}_{t+1}\right)^{2}\right)-1\right)^{\frac{1}{2}}
$$

where $\bar{m}_{t}=\log \bar{M}_{t}$. It also follows from (2.18) and (2.24) that the conditional standard deviation of the logarithm of the stochastic discount factor in the competitive equilibrium is $\sigma_{t}\left(\bar{m}_{t+1}\right)=\sigma_{a}$. Thus, the coefficient of variation of the stochastic discount factor is given by

$$
\frac{\sigma_{t}\left[\bar{M}_{t+1}\right]}{E_{t}\left(\bar{M}_{t+1}\right)}=\left(\exp \left(\sigma_{a}^{2}\right)-1\right)^{\frac{1}{2}}
$$


Having described how to compute the coefficient of variation of the stochastic discount factor, we will see if the relationship between the observable Sharpe ratio and the coefficient of variation of the stochastic discount factor specified in (3.60) holds. Table 2 demonstrates that the observed Sharpe ratio is around 0.5. In addition, as noted earlier, we set $\sigma_{a}=$ 0.00785. Given these parameter values, we can see from (3.60) and (3.62) that in the presence of state contingent claims on consumption goods, allowing for quasi-geometric discounting does not help to modify the lower bound of the coefficient of variation of the stochastic discount factor.

\section{Conclusion}

In this paper, we have investigated the quantitative implications of quasi-geometric discounting for the risk-return trade-off observed in the actual economy. It has been emphasized in recent literature that the current self of a consumer has to rely on its consumption and savings decisions to affect its future self, if conflict between current and future selves exists because of time inconsistent preferences and if commitments on future actions are not feasible. In such an economic environment, consumers can have extra dividends from their asset holdings, which are not paid by issuers of assets. We therefore allow for the possibility that the dividend observed in the financial data may not reflect all the dividend that consumer expect to obtain from their asset holdings, when consumers have greater discount rates in the short-run than in the long-run. We then demonstrate that allowing for such a possibility in stochastic growth models with quasi-geometric discounting may help to give a better understanding of the risk-return trade-off observed in the actual economy without making a large increase in the volatility of the stochastic discount factor. In particular, we have shown that the measure of short-run impatience may not be included in the stochastic discount factor if non-pecuniary payoffs as well as pecuniary payoffs are to be priced in the valuation of assets. Thus, this implies that if the Sharpe ratio is defined in terms of the whole return of an asset, it is not likely to explain the risk-return tradeoff observed in the actual economy, as shown in many consumption-based asset pricing models. We, however, have demonstrated that when both observable and unobservable returns are priced in the absence of state contingent claims, the observable Sharpe ratio can 
match the trade-off between expected return and risk observed in financial data. In sum, our conclusion suggests that allowing for non-pecuniary payoffs would be a mechanism to create the observed magnitude of risk-return trade-offs in consumption-based asset pricing models. 
Table 1

Parameter values

\begin{tabular}{lcl}
\hline Parameter & Values & Description and definitions \\
\hline$\alpha$ & 0.42 & Output elasticity of capital \\
$R$ & 1.0225 & Quarterly average rate of gross return on stocks \\
$\delta$ & 1 & Depreciation rate of capital \\
$H$ & 0.2 & Average fraction of the hours worked \\
$\rho_{a}$ & 0.95 & Persistence parameter for the logarithm of technology process \\
$\sigma_{a}$ & 0.00785 & Standard deviation of technology shocks \\
& & \\
\hline
\end{tabular}


Table 2

Annual real returns on stocks and bonds for 1947 - 1996, U.S.A.

\begin{tabular}{lcccc}
\hline & $\begin{array}{c}\text { Value weighted } \\
\text { NYSE }\end{array}$ & S\&P500 & $\begin{array}{c}\text { Equally weighted } \\
\text { NYSE }\end{array}$ & $\begin{array}{c}\text { 3-month } \\
\text { Treasury bills }\end{array}$ \\
\hline Average return & 9.1 & 9.5 & 11.0 & 0.8 \\
Standard deviation & 16.7 & 16.8 & 22.2 & 2.8 \\
Sharpe ratio & 0.497 & 0.561 & 0.459 & 0 \\
\hline
\end{tabular}

Notes: Average and standard deviation of real returns are percentage.

Sources: Data on average and standard deviation are from Table 1 in Cochrane(1997). 


\section{References}

[1] Cambell, John Y. "Asset Pricing at the Millenium." Journal of Finance, 2000, 55(4), 1515-1567.

[2] Cochrane, John H. "Where is the Market Going? Uncertain Facts and Novel Theories." Economic Perspectives, The Federal Reserve Bank of Chicago, 1997, 21(6), $3-37$.

[3] Hansen, Gary D. "Indivisble Labor and the Business Cylce." Journal of Monetary Economics, 1985, 16, 309-327.

[4] Hansen, Lars Peter and Ravi Jaganathan. "Implications of Security Market Data for Models of Dynamic Economies." Journal of Political Economy, 1991, 99, 225-262.

[5] Harris, Christopher and David Laibson. "Dynamic Choices and Hyperbolic Consumers." forthcoming in Econometrica, 2000.

[6] King, Robert G., Charles I. Plosser and Sergio T. Rebelo. "Production, Growth and Business Cycles I. The Basic Neoclassic Model." Journal of Monetary Economics, 1988a, 21, 195-232.

[7] King, Robert G., Charles I. Plosser and Sergio T. Rebelo. "Production, Growth and Business Cycles II. New Directions." Journal of Monetary Economics, 1988b, 21, 309-342.

[8] Krusell, Per, Burhanettin Kuruscu and Anthony A. Smith. "Equilibrium Welfare Government Policy with Quasi-geometric Discounting." Journal of Economic Theory, 2002, 105, 45-72.

[9] Krusell, Per and Anthony A. Smith. "Consumption-Savings Decisions with QuasiGeometric Discounting." Econometrica, 2003a, 71(1), 365-375.

[10] Krusell, Per and Anthony A. Smith. "Consumption-Savings Decisions with QuasiGeometric Discounting: The Case with a Discrete Domain." Mimeo, University of Rochester, 2003b. 
[11] Lettau, Martin and Harold Uhlig. "Preference, Consumption Smoothing, and Risk Premia." Mimeo, CentER for Economic Research, 1997a.

[12] Lettau, Martin and Harold Uhlig. "Asset Prices and Real Business Cycles: A Diagnostic View." Mimeo, CentER for Economic Research, 1997b.

[13] Laibson, David. "Hyperbolic Discount Functions and Time Preference Heterogeneity." Mimeo, Harvard University, 1997.

[14] Luttmer, Erzo G.J. and Thomas Mariotti. "Subjective Discounting in an Exchange Economy." Journal of Political Economy, 2003, 111(5), 959-989.

[15] Phelps, Edmund. S. and R.A. Pollak. "On Second-best National Saving and Gameequilibrium Growth." Review of Economic Studies, 1968, 35, 185-199.

[16] Pollak R.A. "Consistent Planning." Review of Economic Studies, 1968, 35, 201-208.

[17] Shiller, Robert J. "Consumption, Asset Markets, and Macroeconomic Fluctuations." Carnegie-Rochester Conference Series on Public Policy, 1982, 17, 203-238.

[18] Strotz, Robert H. "Myopia and Inconsistency in Dynamic Utility Maximization." Review of Economic Studies, 1956, 23, 165-180. 


\section{Appendix}

\section{A The Generalized Euler Equation for the Competitive Equilibrium}

Let $L\left(K_{t}, \bar{K}_{t}, A_{t}\right)$ denote the value function for the following dynamic programming problem:

$$
L\left(K_{t}, \bar{K}_{t}, A_{t}\right)=\max _{C_{t}, H_{t}, K_{t+1}} u\left(C_{t}, 1-H_{t}\right)+\beta E_{t}\left[L\left(K_{t+1}, \bar{K}_{t+1}, A_{t+1}\right)\right],
$$

subject to the period budget constraint (2.4) in each period $t=0,1, \cdots, \infty$. Let $\Phi\left(K_{t}, \bar{K}_{t}, A_{t}\right)$ denote the value function of the optimization problem at period $t$ of the representative household. Then, substituting (2.4) into (2.1), one can obtain an expression of $\Phi\left(K_{t}, \bar{K}_{t}, A_{t}\right)$ of the form:

$$
\Phi\left(K_{t}, \bar{K}_{t}, A_{t}\right)=\max _{H_{t}, K_{t+1}} u\left(R_{t} K_{t}+W_{t} H_{t}-K_{t+1}, 1-H_{t}\right)+\theta \beta E_{t}\left[L\left(K_{t+1}, \bar{K}_{t+1}, A_{t+1}\right)\right] .
$$

The first-order conditions for this optimization problem are then given by

$$
\begin{gathered}
u_{1}\left(C_{t}, 1-H_{t}\right)=\theta \beta E_{t}\left[L_{1}\left(K_{t+1}, \bar{K}_{t+1}, A_{t+1}\right)\right], \\
u_{1}\left(C_{t}, 1-H_{t}\right) W_{t}=u_{2}\left(C_{t}, 1-H_{t}\right) .
\end{gathered}
$$

Hence, differentiating $\Phi\left(K_{t}, \bar{K}_{t}, A_{t}\right)$ with respect to $K_{t}$ and substituting (A.3) and (A.4) into the resulting equation yields

$$
\Phi_{1}\left(K_{t}, \bar{K}_{t}, A_{t}\right)=u_{1}\left(C_{t}, 1-H_{t}\right) R_{t}
$$

In addition, substituting (A.1) into (A.2) leads to

$$
\theta L\left(K_{t}, \bar{K}_{t}, A_{t}\right)=\Phi\left(K_{t}, \bar{K}_{t}, A_{t}\right)-(1-\theta) u\left(C_{t}, 1-H_{t}\right)
$$

This in turn implies that, given that the first-order conditions described in (A.3) and (A.4) hold, the partial derivative of $L\left(K_{t}, \bar{K}_{t}, A_{t}\right)$ with respect to $K_{t}$ is

$$
\theta L_{1}\left(K_{t}, \bar{K}_{t}, A_{t}\right)=\Phi_{1}\left(K_{t}, \bar{K}_{t}, A_{t}\right)-(1-\theta) u_{1}\left(C_{t}, 1-H_{t}\right)\left(R_{t}-\frac{\partial K_{t+1}}{\partial K_{t}}\right) .
$$

Therefore, substituting (A.5) into (A.7), one can rewrite (A.7) as follows:

$$
\theta L_{1}\left(K_{t}, \bar{K}_{t}, A_{t}\right)=u_{1}\left(C_{t}, 1-H_{t}\right)\left(\theta R_{t}+(1-\theta) \frac{\partial K_{t+1}}{\partial K_{t}}\right) .
$$


As a result, substituting (A.8) into (A.3), one can obtain an expression of the generalized Euler equation of the form:

$$
1=\beta E_{t}\left[\frac{u_{1}\left(C_{t+1}, 1-H_{t+1}\right)}{u_{1}\left(C_{t}, 1-H_{t}\right)}\left(\theta R_{t+1}+(1-\theta) \frac{\partial K_{t+2}}{\partial K_{t+1}}\right)\right]
$$


Figure 1: The Relationship between the Short-run Impatience and the Unobservable Sharpe Ratio in the Competitive Equilibrium

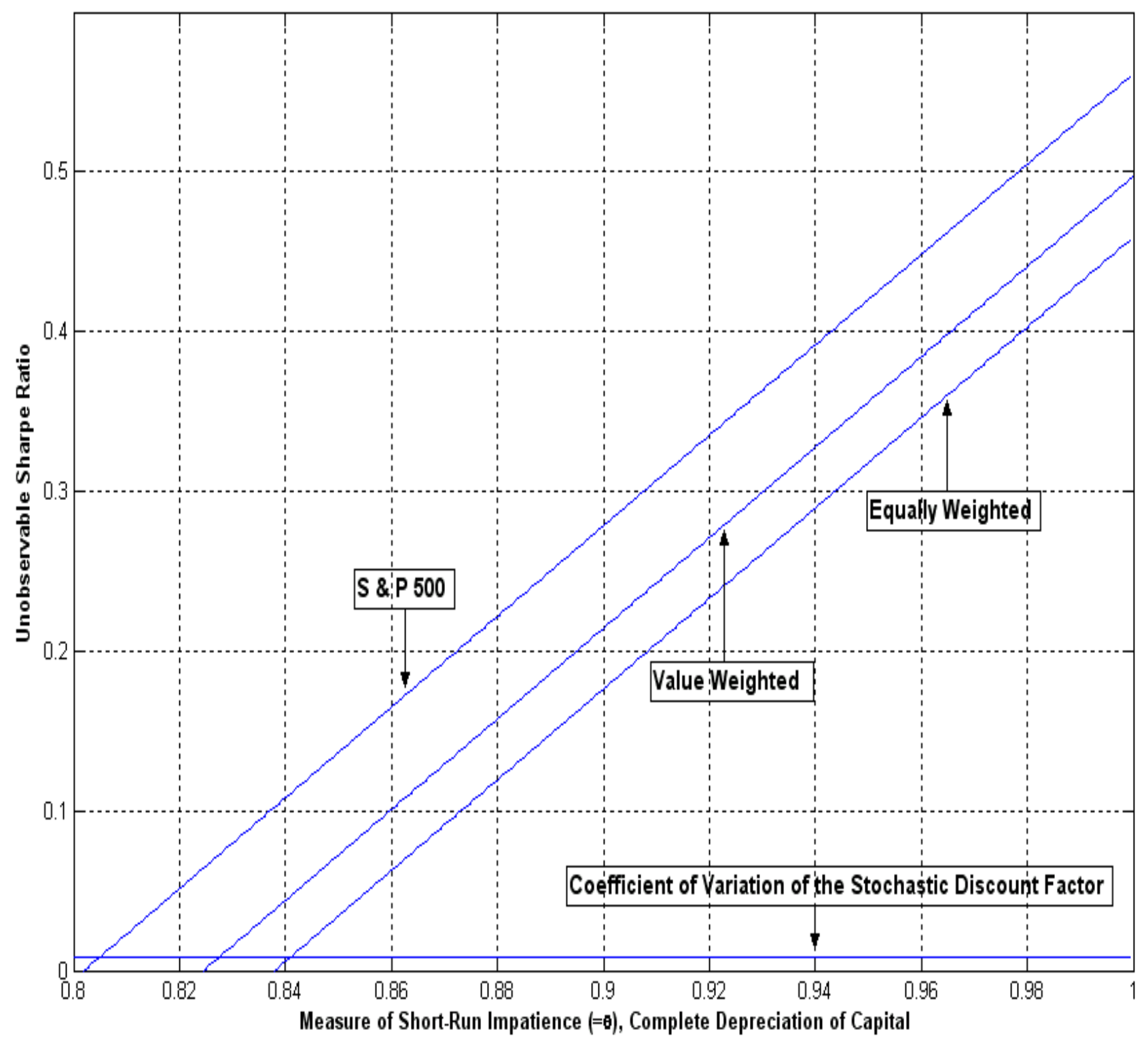


Figure 2: The Relationship between the Short-run Impatience and the Unobservable Sharpe Ratio in the Planning Problem

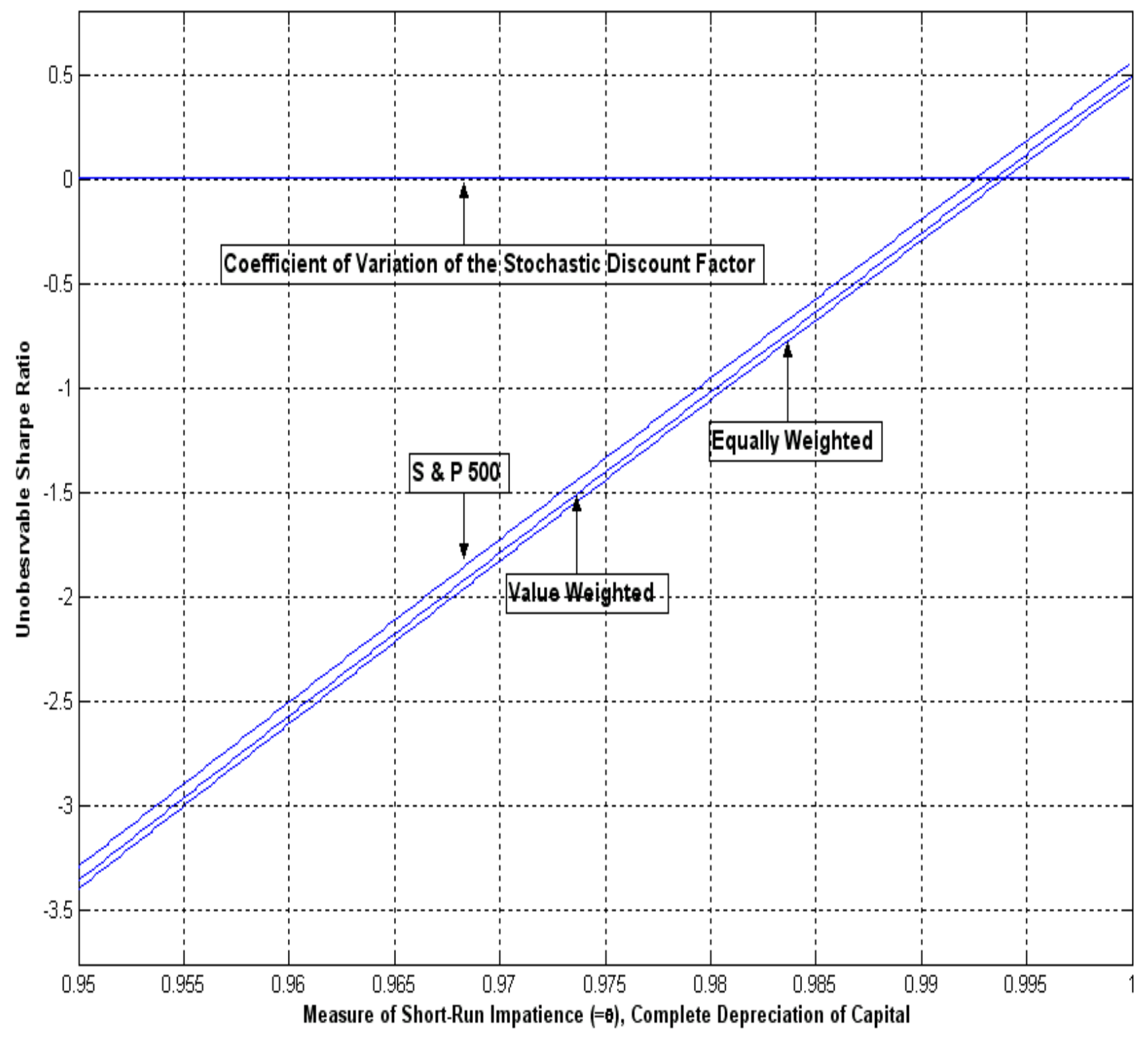

